\title{
Sports Results Measurement and Efficiency in UEFA Champions League
}

\author{
By Fabiola Zambom-Ferraresi ${ }^{*}$ \\ Lucía Isabel García-Cebrián \\ Fernando Lera-López ${ }^{+}$
}

\begin{abstract}
The UEFA Champions League (UCL) is the most important competition at club level. Nevertheless, the sports technical efficiency of this competition has been scarcely analysed. The present paper seeks to fill this gap by analysing from a longitudinal and data panel perspective all the teams that played in the UCL during nine seasons (2004/05 to 2012/13). From a methodological perspective, we have used Data Envelopment Analysis (DEA), a deterministic non-parametric frontier method. In particular, we have applied constant return to scale and variable return to scale DEA assumptions. Due to the tournament characteristics (a group stage in the first phase, and a knockout system in the second phase), we have selected an input-oriented model. As the output (sporting result), we have proposed the use of the coefficients applied by UEFA from UCL revenue distribution. Efficiency is analysed in UCL considering a long period of time and applying the coefficients of revenue distribution as sporting results measurement. On account of differences from previous studies, we are able to obtain some interesting results. First, there is a high inefficiency level in UCL in the analysed seasons. Second, many clubs could be efficient in one season but stop being efficient for all the seasons simultaneously. As a consequence, the teams have many problems maintaining their efficiency during the seasons. Third, we have identified some regular inefficiency sources: waste of resources and inappropriate sporting tactics. Finally, from a longitudinal perspective, it is suggested an improvement in sporting tactics applied by the teams in the last seasons compared with the previous seasons.
\end{abstract}

Keywords: Efficiency, DEA, Football, Champions League, Sports results

\section{Introduction}

Today, football is one of the most important forms of sporting expression, as well as being a business of undoubted economic significance (Palacios-Huerta 2004). Born in Europe, football is already a global phenomenon. Yet it is still dominated by European teams, and the UEFA ${ }^{1}$ Champions League (UCL) remains the most important club-level competition worldwide. For example, the last UCL final, between Real Madrid and Juventus, generated 98 million interactions from 34 million people on Facebook and another 50 million interactions from 14 million on Instagram. In Twitter terms, there were nine million posts relating to the final (UEFA 2017).

The football industry has changed notably over the last two decades and economic survival has become more and more important. According to the

\footnotetext{
* Department of Economics, Public University of Navarra, Spain.

${ }^{\dagger}$ Department of Economics and Business Administration, University of Zaragoza, Spain.

${ }^{+}$Department of Economics, Public University of Navarra, Spain.
} 
Annual Review of Football Finance (Deloitte 2017), total European football market revenues reached almost $€ 25$ billion in the $2015 / 16$ season, a $13 \%$ increase on 2014/15. Nevertheless, these rates of growth cannot continue indefinitely, and although losses have dropped since the full introduction of Financial Fair Play, in 2015 aggregate losses stood at $€ 300 \mathrm{~m}$ (UEFA 2015).

Thus, good utilisation of available resources and a corresponding diminution in costs could be a solution to the problem of losses currently suffered by many football clubs. In a general sense, and in the context of productive processes, efficient organisations are those that either use minimal quantities of inputs in order to obtain outputs or obtain the maximum output from their inputs. Applied to football clubs, the equivalent of productive process are the games played in a championship, while the main causes of sports inefficiency might be the waste of resources or inappropriate tactical choices (Espitia-Escuer and García-Cebrián 2004, 2010, Haas 2003, Torres-Dávila and García-Cebrián 2012)

The main objective of this paper is to determine the efficiency of the football teams that have participated in the UCL in nine seasons (2004/05 to 2012/13), by applying Data Envelopment Analysis (DEA). DEA provides a single measure of efficiency when dealing with multiple inputs and outputs. This methodology can also supply useful information about the sources of clubs' inefficiency. Thus, this research could contribute to the better use of sports resources by clubs as a way of reducing economic costs.

In this paper we pose two research questions. The first concerns the level of efficiency that UCL football clubs have demonstrated in the period 20042013. Although there is extensive literature on football efficiency measured through DEA, for national leagues (Haas 2003, Espitia-Escuer and GarcíaCebrián 2004, 2006, Barros and Leach 2006, Barros and Douvis 2009, Boscá et al. 2009, González-Gomez and Picazo-Tadeo 2010, Ribeiro and Lima 2012), few studies have analysed European competitions (Papahristodoulou 2006, EspitiaEscuer and García-Cebrián 2010). Nevertheless, these studies show some limitations in the selection of the sport outputs and analyse a limited number of seasons. On the basis of previous empirical evidence, we would expect a low efficiency level among European football clubs.

The second research question concerns the most important causes of being inefficient. On this question, the results of national studies diverge. On the one hand, Espitia-Escuer and García-Cebrián (2004), and Torres-Dávila and GarcíaCebrián (2012) observed that Spanish and Mexican teams suffer from a poor choice of technology (i.e. wrong tactics used). On the other, Hass (2003) and Espitia-Escuer and García-Cebrián (2010) found, respectively, that themain cause of inefficiency in English Premier League clubs and teams playing in the UCL was their waste of resources. Because our sample size is larger than those of previous studies, we hypothesise that we will find a greater range of causes behind UCL clubs' inefficiency.

This study contributes to previous research in several ways. First, we use a measure of sports output that aims to overcome certain problems detected in previous studies. This output measure is the revenue obtained, according to the coefficients applied by UEFA, from UCL revenue distribution on the basis of 
sporting performance. This output measure is more representative and reliable than measures used in previous studies (Espitia-Escuer and García-Cebrián 2010, González-Gomez and Picazo-Tadeo 2010, Papahristodoulou 2006, TorresDávila and García-Cebrián 2012).

Second, to carry out our approach our sample from the individual seasons of 2004/05 to 2012/13 was analysed as a whole. In this way, we overcame one of the limitations often attributed to DEA, which is its sensitivity to the sample size used. This more robust analysis provides accurate, objective and relevant information. This can help the decision-making of coaches and managers, and, as a consequence, can lead to improvements in football clubs' efficiency. Furthermore, the differences between the efficiency results obtained from the season-by-season analysis and that of the sample as a whole, throw up useful recommendations for participating UCL teams.

The remainder of this article is structured as follows. The next section briefly introduces efficiency' concepts, the football teams' production process and a literature review. The third section describes the methodology and explains the output and input variables used. Results are presented in section four. The last section discusses the results and concludes this paper.

\section{Frameworks and Literature Review}

\section{Efficiency and Production Function in football}

According to Debreu (1951), efficiency is an economic term related to the good use of inputs, in the sense that an organisation is efficient if it obtains the maximum possible output or it uses the minimum possible input. With the aim of empirically calculating efficiency, Farrell (1957) proposed the distance to isoquant as a measurement of efficiency for organisations or DMUs (decisionmaking units). Hence, estimation of the isoquant becomes the key point. For this purpose, Farrell (1957) suggests the use of real data inputs and outputs of a sample composed of homogenous organisations, belonging to the same economic sector at least. The estimated isoquant, called efficient frontier by Farrell (1957), is formed by best-practice DMUs in the sample. As a consequence, a sound knowledge of productive function is necessary to establish the variables representative of inputs and outputs.

In the case of football teams, efficiency means the maximisation of sports results (goals, wins or points) with the actual number of physical resources, or the minimisation of resources to obtain the actual sport result. In terms of the production function of sports teams, Scully (1974) was the first to analyse this, which he did for baseball. Works such as Schofield (1988) on cricket, Carmichael and Thomas (1995) on rugby, and Carmichael et al. (2000) on football, consider that production function in sports is composed of two different stages. More recent studies, specifically about football, corroborate that the production function is composed of two different stages, each with its own inputs and outputs (Espitia- 
Escuer and García-Cebrián 2004, 2006, 2010, Torres-Dávila and García-Cebrián 2012).

In figure 1 we can see the production process flow (from left to right). A squad and a coaching staff (input 1), with their skills and characteristics, will carry out some attack and defence plays (output 1 and input 2) which will produce a result (output 2). In an isolated match, this result can be goals or a win, or, in a league or a tournament, points or progress to the next phase. In breaking down this production process we may observe two stages:

- In the first stage players and coach, through their pre-match work (technical, tactical and physical workouts), will train to produce attack and defence plays during the match.

- In the second stage these plays, which are the output from the first stage, will be the input and generate an outcome - goals, wins, points, etc. depending on the context.

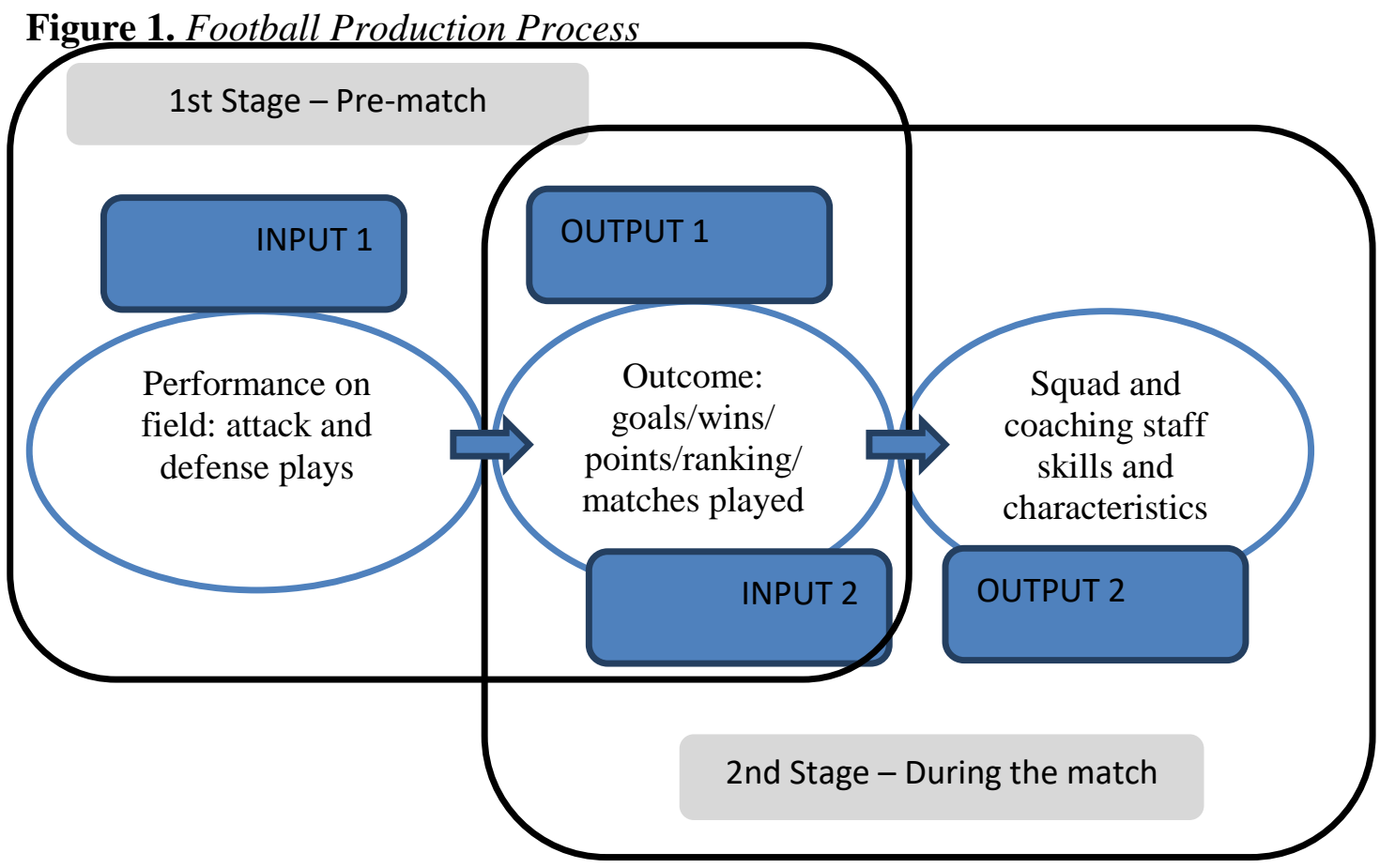

Source: Authors' elaboration adapted from Espitia-Escuer and García-Cebrián 2004.

\section{Literature Review}

A review of the existing empirical evidence on efficiency in football teams shows differences in the methodologies applied, the variables analysed (sports measures) and the units under analysis (the manager, the coach or the club as a whole).

Although in their survey of efficiency measurement tools, Førsund, Lovell and Schmidt (1980) identified four methodologies, the most commonly used since publication of their study have been parametric stochastic frontiers and 
deterministic non-parametric frontiers (since called DEA). On one hand, the parametric stochastic approach needs to specify the functional form for the production function that relates the inputs to the outputs, and does not enable analysis with multiple outputs. Dawson et al. (2000) analysed the efficiency of managers in the English Premier league using an econometric stochastic frontier, while Carmichael et al. (2000) and Barros and Garcia-de-Barrio (2008) employed stochastic frontier models to analyse the efficiency of English football clubs. On the other hand, the deterministic non-parametric approach does not need the production function to be specified and has been widely applied to measure efficiency in football clubs (Barros and Douvis 2009, Barros and Leach 2006, Boscá et al. 2009, Espitia-Escuer and García-Cebrián 2004, 2006, 2010, González-Gómez and Picazo-Tadeo 2010, Haas 2003, Papahristodoulou 2006, Ribeiro and Lima 2012).

In terms of the variables employed, Carmichael et al. (2000), Espitia-Escuer and García-Cebrián $(2004,2006)$ and Torres-Dávila and García-Cebrián (2012) have estimated the sports efficiency of football clubs using match statistics of the games played as inputs and the final ranking or points in the league as outputs. Boscá et al. (2009) also used match statistics as their input, but their output variables were goals scored and conceded; they separately analysed and compared the offensive and defensive efficiency of the Italian and Spanish leagues. Where the tournament is of a mixed nature, consisting of a group stage and a knockout phase, there is no consensus on what output variables to apply. For example, Haas (2003a) analysed efficiency in Major Soccer League, (American- Canadian league) and used the points achieved in the regular league and the wins in the knockout phases as output. González-Gómez and Picazo-Tadeo (2010) observed the three tournaments that Spanish clubs play; the output to the league was the points achieved, the number of rounds played to win the cup, and the number of games played in European competitions (UCL and UEFA Europa League). Torres-Dávila and García-Cebrián (2012) used the points in the group stage and the games played in the knockout phase to study separately the Mexican national league phases. Finally, Papahristodoulou (2006) and Espitia-Escuer and García-Cebrián (2010) used the number of games played to evaluate the efficiency of UCL. This number represents teams' progress in competition, which is the main objective in this competition type. A team that has reached the round of 16, for example, has had an output of eight games, six in the group stage and two in the second round.

Although the concept of efficiency is focused on production processes, other streams of analysis have looked at financial measures. Authors such as Gerrard (2010) and Ribeiro and Lima (2012) have used sports results as output and measures of financial expenditure (i.e. wages) as input. In a similar vein, Giner-Vicente and Muñoz-Porcar (2008) employed six different indicators of financial expenditure, the club's final ranking and revenue as output. GonzálezGómez and Picazo-Tadeo (2010) included two traditional measures as inputs: number of seasons in the first division and number of trophies won in national and international competitions. 
Previous research can also be classified according to the geographical area under study. Traditionally, studies have adopted a national approach, the national leagues most studied being the English Premier League (Barros and Leach 2006, Carmichael et al. 2000, Gerrard 2010, Haas 2003) and the Spanish Liga (Boscá et al. 2009, Espitia-Escuer and García-Cebrián 2004, 2006, Giner-Vicente and Muñoz-Porcar 2008, González-Gómez and Picazo-Tadeo 2010). However, less well-known leagues have also been analysed: the Portuguese (Barros and Douvis 2009, Ribeiro and Lima 2012); the Italian (Boscá et al. 2009); the Greek League (Barros and Douvis 2009); the Major Soccer League of North America (Haas 2003a); and, the Mexican League (Torres-Dávila and García-Cebrián 2012).

Nevertheless, studies looking at international competitions and the UCL in particular, are scarce. Papahristodoulou (2006) analysed the UCL in five different sets of combinations of inputs and outputs; in three of them the analysis was focussed just on the UCL group stage, and in another two he observed the competition as a whole. Three clubs were efficient in all combinations of inputs and outputs used. However, the efficiency rankings and the efficiency scores changed when the author changed the combination of variables.Espitia-Escuer and García-Cebrián (2010) obtained interesting results. They concluded that efficiency could be used to evaluate positively those teams that achieve the results their potential allows them, and to recognise which teams obtain good results although they waste resources. Furthermore, from a pooled analysis of all the seasons, they concluded that there are no different dominating tactics, but there is a general waste of resources in some of the seasons.

\section{Methodology and Data}

\section{Methodology}

Calculations for measuring efficiency using the deterministic non-parametric frontier method were proposed by Charnes et al. (1978), who were the first to use the term Data Envelopment Analysis (DEA). These calculations involve solving linear programming problems in order to construct a non-parametric frontier over the data. Their basic model, known as the CCR model (Charnes, Cooper and Rhodes), can process non-discriminatory variables. The CCR model assumes constant return to scale (CRS) and calculates the technical efficiency (TE) scores of the DMUs. This model may be oriented towards the input or the output. Therefore, the efficiency of each DMU of the sample consists in solving the following linear programming problem:

$$
\begin{aligned}
& \operatorname{Min}_{\lambda z} \lambda_{1 i} \\
& \text { s.t. } u_{i} \leq z_{i} U \\
& \lambda_{1 i} x_{i} \geq z_{i} X \\
& z \in R_{+}^{k}
\end{aligned}
$$


where $\lambda_{1 i}$ is the technical efficiency index considering an orientation to the input; $u_{i}$ is the vector that represents the quantities of $m$ products produced by the organisation $i$; $U$ is the matrix of range $k . m$ which represents the quantities of $m$ products for the $k$ organisations in the sample; $x_{i}$ are the quantities of the $n$ production factors used by the organisation whose efficiency is being measured; $X$ is the matrix of range $k . n$ for the quantities of $n$ production factors used by the organisations in the sample; and $z_{i}$ is an intensity parameters vector that determines the combinations of factors and products observed. The technical efficiency value indicates the radial reduction that could be implemented in the consumption of production factors if the unit studied were to become efficient. When $\lambda_{1 i}=1$, the organisation analysed belongs to the frontier; it is impossible to obtain its production vector with a radial reduction of all its resources and it is classified as an efficient unit. This is the maximum value that the efficiency of an organisation can take. If $\lambda_{1 i}<1$, this indicates the proportion to which the quantity of all inputs used to achieve at least the actual output quantity could be reduced radially, so the output will be obtained without wasting resources.

Banker et al. (1984) proposed some adjustments to the CRS model in which variable returns to scale (VRS) are assumed. Their model, known as BCC (Banker, Charnes and Cooper), allows calculating the pure technical efficiency (PTE). The use of the VRS specification is suggested for an efficiency calculation when not all DMUs are operating at the optimal scale. The linear programming problem to solve in this case forms the reference unit from units of a similar size/technology, ${ }^{2}$ which leads to the following formula

$$
\begin{aligned}
& \operatorname{Min}_{\lambda, z} \lambda_{2 i} \\
& \text { s.t. } u_{i} \leq z_{i} U \\
& \lambda_{2 i} X \geq z_{i} X \\
& \Sigma z_{1}=1 \\
& z \in R_{+}^{k}
\end{aligned}
$$

where $\lambda_{2 i}$ is the pure technical efficiency of the unit studied. The pure technical efficiency value indicates the radial reduction in the consumption of production factors that the unit studied should implement in order to be efficient when compared with those units of similar technology. In the case of football teams, tactics developed during games are the equivalent to technology. Therefore, if we have $\lambda_{1 i}<1$ and $\lambda_{2 i}=1$, this indicates an appropriate use of the resources, without waste, and the inefficiency is due to the wrong tactics being chosen.

The scale efficiency (SE) is given by the ratio between the TE score and the PTE score. Generally, if $\mathrm{SE}=1$ it means that the DMU is operating at an optimal scale. If $\mathrm{SE}<1$, the DMU is not working at the most productive scale size (technology or tactics for football teams).

Depending on the data availability, the DEA methodology could be applied in different ways (Tulkens and Vanden Eeckaut 1995, Asmild et al.

\footnotetext{
${ }^{2}$ Normally, in DEA literature the size of a unit is related to the different technologies that could exist to make a product. When the term "size" is employed in the present paper, we are making a reference to the traditional "financial-economic" size of the football clubs, like regular companies.
} 
2004, and Culliane and Wang 2006). For example, when we have a data panel with different observations in several seasons for the same teams, it is possible to develop a static analysis. In this case, efficiency for each team is calculated in every season considering exclusively as sample the different observations in that season. On the other hand, a dynamic analysis could be developed. In the easier version, this dynamic analysis supposes the estimation of the efficiency for each team in every season, but considering the sample as a whole.

In the present paper, efficiency values during all the seasons for each team were estimated using the whole sample. From a methodological approach, in the calculation of efficiency taking into account all the seasons of the sample as a whole, the efficiency of each particular team will never increase versus that obtained in a season-by-season analysis. DEA is the resolution of linear programming problems, and its mathematical property is that the optimal never increases when the number of constraints increases. This is what happens when expanding the sample, and is, precisely, one of the most important advantages of the dynamic analysis. As the results obtained with the DEA are very dependent on the used sample, when the sample is higher, the results obtained are more robust. Then, when all the seasons are considered, simultaneously, it could be argued that the efficient teams really have made an adequate use of sport resources.

Furthermore, the results obtained with the sample as a whole allow developing a more rigorous evaluation of efficiency. The differences among the efficient rates calculated in different seasons for a team could be as a consequence of changes in the resources used by the team or differences in the sample from one season to another. On the contrary, when the estimations are made considering all the seasons as a whole, the second argument disappears. Consequently, in this case, differences in efficiency rates for a team could be exclusively due to the selection of the sport resources (amount of resources and their use in specific strategies and tactics).

In this research we calculated technical efficiency, pure technical efficiency and scale efficiency. They are well-known and have been widely used in the last twenty years in many areas (public universities, banks, public sector resources, sports, etc.). These models have also been used in football (Haas, 2003; Barros and Leach 2006, Espitia-Escuer and García-Cebrián 2004, 2010, Torres-Dávila and García-Cebrián 2012).

\section{Data and Variables}

The UCL is Europe's top international football competition at club level. Initially contested by the champions of the national leagues, it now has several qualifying routes. The UEFA coefficient system $^{3}$ is used to set criteria for direct access into the group stage or access to the previous qualifier rounds. The competition itself starts at the group stage, with eight groups of four teams in each. A team plays twice each of the others in its group, in matches as host

\footnotetext{
${ }^{3}$ More information about the competition format and the UEFA coefficient system is available at: http://www.uefa.com/memberassociations/uefarankings/index.html (accessed 18 February 2014).
} 
and visitor. The two highest ranked teams in every group advance to the next stage. With 16 teams qualifying for the second round, the knockout stage is characterised by double matches, home and away, with the winner advancing to the next phase, and so on until the semi-final. The final, exceptionally, is played as a single match, at a ground chosen the previous year. The UCL champion qualifies to compete for the European Super Cup and the FIFA Club World Cup. Our intention was to analyse group stages and knock-out rounds together, in accordance with Espitia-Escuer and García-Cebrián's (2010) approach.

The sample in this article consists of the 32 clubs that qualified for the UCL group stage in each of the nine seasons between 2004/05 and 2012/13: a total of 288 units of observation. Some of these 288 DMUs are the same club in different seasons. In total, there are 94 different clubs. Many clubs participated only once in the competition during the study period, but a select group consisting of Arsenal, Barcelona, Chelsea, Manchester United and Real Madrid participated in all nine of the seasons.

The performance on field, that is the outcome of actions taken by teams during the match, expressed through movements of attack and defence, is the core of football clubs production process. As can be seen in the framework, these attack and defence plays are the output of the production process first stage (Figure 1), but they are also the input of the second stage. The present study is focussed on the production process second stage, so the sports variables are our inputs. Following previous literature, total attempts, ball possession and ball recoveries are the selected inputs variables and the details of the reasons for this choice will be set out in what follows.

The total attempts made are the completion of the offensive plays of a team. Lago-Peñas et al. (2011) analysed group stage matches of three UCL seasons $(2007 / 08-2009 / 10)$, and their results showed that winning teams had average values significantly higher $(\mathrm{p}<0.01)$ for total shots than losing teams. TorresDávila and García-Cebrián (2012) used it as an input measurement.

The minutes of ball possession represent the volume of play of a team, which usually indicates which team has dominated the match, or has shown more initiative and intent to have the ball. Lago-Peñas et al. (2011) concluded that ball possession is an indicator of success in UCL. Espitia-Escuer and García-Cebrián (2006, 2010) and Papahristodoulou (2006) used it in their models.

Ball possession could have potential limitations in the development of tactics in opposition sports. A team that plays a counter-attack may not take the initiative to possess the ball, counting instead on the speed and capacity of its players. Possibly, therefore, it creates less chances of plays completion (minus attempts). However, it will usually generate more situations of numerical superiority, a big advantage in football. All this would mean that such a team, with little ball possession and few attempts on target, becomes fairly efficient, if it manages to turn its counter-attacks into goals. Then, in order to overcome this potential limitation, ball recovery is an important indicator of that active attitude in relation to controlling the match. Furthermore, this variable can be a substitutive input, because if a team has much ball possession, consequently, it 
will have fewer opportunities to recover the ball, and vice versa. Therefore, a ball recovery, a defensive play, is the third input variable used in the model.

If there is a point at which there is consensus in the literature, it is that the output variable must be sporting performance, or one of them, in the case of more than one output. In national regular leagues the most common way to measure this outcome is through the points archived (Haas 2003, Espitia-Escuer and GarcíaCebrián 2004, 2006, Barros and Leach 2006, González-Gómez and PicazoTadeo 2010). However, there are more ways to measure it; for example, GinerVicente and Muñoz-Porcar (2008) and Garcia-del-Barrio and Szymanski (2009) used the final league ranking, while Carmichael and Thomas (1995) and Horowitz (2007) used wins percentage over total games played.

One of the contributions of this paper is the output variable used for calculating efficiency: first, because most of the literature is focused on championships played as leagues (single or double round-robin tournaments); and, second, because the usual output variable employed to measure efficiency in knock-out tournaments is the number of games played. In the UCL case, if we use the number of games played, all the clubs involved in the group stage would get the same output, regardless of whether they had won, tied or lost their respective matches. The same happens with the finalists: the winning team and the runners-up obtain the same result, since they play the same number of matches. Using the number of games played, all 16 teams that do not qualify for the knockout stage would get the same result, regardless of their performance, and, consequently, this would lead to bias in the performance evaluation. To solve the problem of the measurement of sporting results in knockout competitions, we propose the use of the coefficients applied by UEFA for the UCL revenue distribution. These coefficients are the output variable used in the present paper, and appear to be a more accurate and representative way of measuring the output of football clubs that participate in competitions like the UCL with a mixed format. This output measure fully preserves the order of the final ranking in the competition, overcoming some limitations shown by previous studies. A team that qualifies to an advanced phase never has a lower score than an eliminated team, and, furthermore, the scale according to sports performance distinguishes it, even though it was eliminated in the same stage of the tournament.

The distribution of UCL revenues is as follows. A fixed part of the amount of revenue ${ }^{4}$ from media rights and commercial contracts is allocated to clubs and corresponds to the sports results achieved. The group stage participation and performance are rewarded, adding a bonus for wins or draws. At the knockout stage they are rewarded with a pass to the next phase. The other part

\footnotetext{
${ }^{4}$ UEFA negotiates agreements with sponsors and television in three-year cycles. Between $75 \%$ and $82 \%$ of the total revenue from media rights and commercial contracts concluded by UEFA goes to the clubs, while the remaining $18 \%-25 \%$ is reserved for European football, for UEFA to cover its organisational and administrative costs and to make solidarity payments to associations, clubs and leagues. What defines the exact value of these figures is the revenue for each period; for the season of $2012 / 13$, for example, up to a maximum of $€ 530 \mathrm{~m}, 75 \%$ of the total was paid over to the clubs, and any revenue in excess of this value raised the clubs' percentage to $82 \%$.
} 
concerning revenue is variable, depending on the market pool, and is not related to sports performance. Therefore, it is not relevant to this study. For all analysed periods the prizes respect the same criteria (as can be seen in Appendix 1, a table summarising prize values evolution). Participation in each phase and the performance in a group stage are rewarded. As the contracts with sponsors and television are negotiated by UEFA in three-year cycles, the prize values also change in the same cycles.

For instance, in the 2012/13 UCL each of the 32 clubs involved in the group stage received a base fee of $€ 8.6 \mathrm{~m}$, and in terms of performance a bonus of $€ 1 \mathrm{~m}$ was paid for a win and $€ 500.000$ for a draw. Teams which qualified for the round of 16 received $€ 3.5 \mathrm{~m}$, the quarter-finalists $€ 3.9 \mathrm{~m}$ and the semifinalists $€ 4.9 \mathrm{~m}$. The UCL winners won $€ 10.5 \mathrm{~m}$ and the runners-up $€ 6.5 \mathrm{~m}$. So, for this season, the minimum a club received for its sports performance in $2012 / 13$ could be $€ 8.6 \mathrm{~m}$ and the maximum $€ 37.4 \mathrm{~m}$, depending on its sports performance. For other seasons, the minimum and maximum values are shown in the Appendix. Thus, the problem outlined above is solved; furthermore, to differentiate the champion team from the runners-up, the proposed measure also best represents the results obtained in the group stage.

To analyse the efficiency during all the seasons, we should consider the entire sample as a whole. Estimating the efficiency of the entire sample as a whole makes it possible to know the best practices in terms of tactics and use of resources. Namely, a club could be efficient in a specific season, considering the power and the resources employed by its opponents. However, if it employed the same resources in the same way in another season, it may not be enough, or this club could waste its resources. Similarly, in knockout competitions, a club could match stronger or weaker opponents. As a consequence, the team will need to employ its resources in different combinations and amounts depending on the opponent characteristics. Therefore, it is important to analyse the efficiency of the sample as a whole. As the values of revenue distribution applied by UEFA changes in a three-year cycle, we should homogenise them. The proposed output measure is a monetary value that represents the sporting results, so we consider the coefficients values of the last season analysed (2012/13) as reference and they have been applied to the other seasons. Moreover, in order to make results comparable, these homogenised values were also used in the season-by-season analysis. In the following, we will make some interesting comparisons.

The descriptive statistics for the inputs and output variables is shown in Table 1. The sports data used in this study were generously provided by Opta Sports, a company with one of the largest sports databases of European football. The UCL results were consulted in the official UEFA website ${ }^{5}$.

\footnotetext{
${ }^{5}$ http://www.uefa.com.
} 
Vol. 4, No. 4 Zambom-Ferraresi et al.: Sports Results Measurement and Efficiency...

\begin{tabular}{|c|c|c|c|c|c|}
\hline & \multicolumn{5}{|c|}{ Table 1. Des criptive Statis tics of the Data Used } \\
\hline & & Total attempts & s Ball recoveries & Ball possession & Sports results \\
\hline \multirow[t]{4}{*}{ UCL 2004-05 } & $\operatorname{Max}$ & 168 & 690 & 375 & 34.9 \\
\hline & Min & 46 & 210 & 119 & 8.6 \\
\hline & Average & 100.44 & 373.59 & 209.63 & 15.47 \\
\hline & SD & 38.59 & 135.31 & 69.77 & 6.60 \\
\hline \multirow[t]{4}{*}{ UCL 2005-06 } & $\operatorname{Max}$ & 217 & 748 & 390 & 36.9 \\
\hline & Min & 51 & 234 & 132 & 8.6 \\
\hline & Average & 103.28 & 404.81 & 200.47 & 15.47 \\
\hline & SD & 41.42 & 136.12 & 66.73 & 6.87 \\
\hline \multirow[t]{4}{*}{ UCL 2006-07 } & Max & 209 & 750 & 352 & 34.9 \\
\hline & Min & 50 & 274 & 129 & 8.6 \\
\hline & Average & 105.56 & 427.19 & 197.34 & 15.47 \\
\hline & $\mathrm{SD}$ & 41.73 & 137.53 & 62.70 & 6.50 \\
\hline \multirow[t]{4}{*}{ UCL 2007-08 } & $\operatorname{Max}$ & 216 & 813 & 401 & 36.9 \\
\hline & Min & 44 & 278 & 128 & 8.6 \\
\hline & Average & 107.34 & 436.69 & 204.41 & 15.47 \\
\hline & $\mathrm{SD}$ & 45.59 & 138.08 & 71.67 & 6.78 \\
\hline \multirow[t]{4}{*}{ UCL 2008-09 } & $\operatorname{Max}$ & 227 & 769 & 428 & 35.9 \\
\hline & Min & 49 & 298 & 117 & 9.1 \\
\hline & Average & 105.06 & 435.72 & 199.84 & 15.47 \\
\hline & $\mathrm{SD}$ & 45.48 & 136.20 & 78.58 & 6.62 \\
\hline \multirow[t]{4}{*}{ UCL 2009-10 } & $\operatorname{Max}$ & 204 & 695 & 445 & 34.9 \\
\hline & Min & 34 & 243 & 118 & 8.6 \\
\hline & Average & 102.84 & 408.47 & 195.94 & 15.47 \\
\hline & SD & 44.63 & 135.92 & 80.45 & 6.57 \\
\hline \multirow[t]{4}{*}{ UCL 2010-11 } & Max & 221 & 984 & 504 & 36.4 \\
\hline & Min & 45 & 364 & 119 & 8.6 \\
\hline & Average & 105.44 & 540.41 & 205.44 & 15.47 \\
\hline & $\mathrm{SD}$ & 46.55 & 164.32 & 84.70 & 6.91 \\
\hline \multirow[t]{4}{*}{ UCL 2011-12 } & $\operatorname{Max}$ & 255 & 994 & 472 & 35.4 \\
\hline & Min & 54 & 340 & 121 & 8.6 \\
\hline & Average & 107.50 & 546.59 & 206.97 & 15.47 \\
\hline & SD & 52.17 & 172.35 & 83.84 & 6.70 \\
\hline \multirow[t]{4}{*}{ UCL 2012-13 } & $\operatorname{Max}$ & 231 & 960 & 472 & 35.9 \\
\hline & Min & 42 & 344 & 99 & 9.1 \\
\hline & Average & 103.19 & 534.53 & 208.13 & 15.47 \\
\hline & $\mathrm{SD}$ & 48.01 & 163.86 & 77.57 & 6.75 \\
\hline
\end{tabular}

Notes: UCL: UEFA Champions League; Max: maximum; Min: minimum; SD: standard deviation

\section{Results}

The results of the pooled analysis are shown in Table 2 sorted by TE score. Following the methodology described in the previous section, the results will be introduced in a similar approach. First, it is shown the technical efficiency results, emphasising the more efficient seasons and clubs. Second, pure technical efficiency results are explained, allowing us to identify the clubs that did not waste their resources and where inefficiency was exclusively due to wrong choice of tactics. Finally, the results considering scale efficiency are shown. 
Table 2. Efficiency Values for the whole Sample

\begin{tabular}{|c|c|c|c|c|c|c|c|}
\hline DMU & TE & PTE & SE & DMU & TE & PTE & SE \\
\hline APOELC1 & 1,00 & 1,00 & 1,00 & CelticC08 & 0,82 & 0,8 & 0,92 \\
\hline CFR & 1,00 & 1,00 & 1,00 & Internazionale $\mathrm{C} 0$ & 0,82 & 0,8 & 0,98 \\
\hline InternazionaleC10 & 1,00 & 1,00 & 1,00 & Paris Saint-GermainC05 & 0,82 & 0,9 & 0,88 \\
\hline LiverpoolC05 & 1,00 & 1,00 & 1,00 & RangersC06 & 0,82 & 0,9 & 0,90 \\
\hline APOELC1 & 0.99 & 1,00 & $\mathbf{0 . 9 9}$ & Atlético MadridC09 & 0,81 & 0,8 & 0,93 \\
\hline Manchester UnitedCO8 & 0,98 & 1,00 & 0,98 & JuventusC09 & $\mathbf{0 , 8 1}$ & $\mathbf{0 , 8 9}$ & $\mathbf{0 , 9 1}$ \\
\hline BarcelonaC06 & 0,97 & 0,99 & 0,98 & CelticC13 & 0,81 & 0,8 & 0,99 \\
\hline BarcelonaC09 & 0,96 & 0,97 & 0,99 & LyonC06 & 0,81 & 0,8 & 0,98 \\
\hline OlympiacosC 0 & 0,96 & 1,00 & 0,9 & ZürichC1 & 0,81 & 0,9 & 0,81 \\
\hline Maccabi Tel AvivC05 & 0,95 & 1,00 & 0,9 & Manchester UnitedC09 & 0,80 & 0,8 & 1,00 \\
\hline Dynamo & 0,92 & 1,00 & 0,9 & VillarrealC0 & 0,80 & 0,8 & 0,99 \\
\hline ChelseaCl2 & 0,92 & 0,96 & 0,96 & InternazionaleC0 & 0,80 & 0,8 & 0,98 \\
\hline ArsenalC0 & $\mathbf{0 . 9 2}$ & $\mathbf{0 . 9 2}$ & 1,00 & MalagaC13 & 0,80 & 0,8 & 1,00 \\
\hline MilanCo7 & 0,92 & 0,92 & $\overline{1,00}$ & ArtmediaC0 & 0,80 & 0,9 & 0,84 \\
\hline ChelseaC0 & 0,92 & 0,92 & 0,9 & Schalke 04C11 & 0,80 & $\mathbf{0 , 8 0}$ & $\mathbf{0 , 9 9}$ \\
\hline LiverpoolCo & $\mathbf{0 . 9 1}$ & $\mathbf{0 . 9 2}$ & 1,00 & OlympiacosC 0 & 0,80 & 0,86 & 0,92 \\
\hline MilanC0 & 0,9 & 0,9 & 1,0 & Sporting LisboaC09 & $\mathbf{0 . 7}$ & $\mathbf{0 . 8 9}$ & $\mathbf{0 . 8 9}$ \\
\hline FenerbahçeC0 & 0,9 & 0,9 & 0,9 & Manchester UnitedC11 & $\mathbf{0 . 7}$ & $\mathbf{0 . 7 9}$ & 1,00 \\
\hline Bayern & 0,9 & 0,9 & 1,0 & ValenciaC0 & 0,79 & 0,95 & 0,84 \\
\hline CSKA & 0,8 & 0,9 & 0,9 & Bayern MunichC13 & 0,79 & 0,83 & 0,96 \\
\hline PortoC0 & 0,8 & 0,9 & 0,9 & LyonC1 & 0,79 & 0,82 & 0,96 \\
\hline PSV EindhovenC06 & 0.89 & $\mathbf{0 . 9 7}$ & $\mathbf{0 . 9 1}$ & OlympiacosC1 & 0,79 & 0,85 & 0,94 \\
\hline Bayern & 0,8 & 0,9 & 0,9 & NapoliC1 & 0,79 & 0,79 & 1,00 \\
\hline Manchester UnitedC05 & 0,8 & 0,9 & 0,9 & LiverpoolC1 & 0,79 & 0,83 & 0,95 \\
\hline Borussia DortmundC13 & 0.8 & 0,8 & 0,9 & BarcelonaC0 & 0.79 & 0,79 & 0,99 \\
\hline Panathinaikos $\mathrm{C} 0$ & 0,8 & 0,9 & 0,9 & ChelseaC1 & 0,79 & 0,81 & 0,97 \\
\hline InternazionaleC0 & 0,8 & 0,9 & 0,9 & BenficaC07 & 0,79 & 0,90 & 0,88 \\
\hline Schalke & 0,8 & 0,9 & 0,9 & $\mathrm{AjaxC0}$ & 0,79 & 0,81 & 0,97 \\
\hline BordeauxC1 & 0,8 & 0,8 & 0,9 & MilanC06 & 0,79 & 0,79 & 0,99 \\
\hline Shakhtar DonetskC05 & 0,8 & 0,9 & 0,9 & ValenciaC13 & $\mathbf{0 . 7}$ & $\mathbf{0 . 8 2}$ & $\mathbf{0 . 9 6}$ \\
\hline Werder & 0,8 & 0,8 & 0,9 & Sporting BragaC11 & 0,78 & 0,85 & 0,92 \\
\hline PortoC0 & 0,8 & 0,8 & 0,9 & ChelseaC0 & 0,78 & 0,78 & 1,00 \\
\hline AnorthosisC0 & 0,87 & 1,00 & 0,87 & CelticC07 & 0,78 & 0,84 & 0,92 \\
\hline Rubin & 0,8 & 0,9 & 0,8 & SevillaC10 & 0,78 & 0,79 & 0,98 \\
\hline Slavia & 0.86 & $\mathbf{0 . 9 7}$ & 0.88 & MilanC10 & 0,77 & 0,80 & 0,97 \\
\hline BarcelonaC11 & 0,86 & 0,87 & 0,99 & OlympiacosC1 & 0,77 & 0,8 & 0,93 \\
\hline ArsenalC13 & $\mathbf{0 . 8 5}$ & $\mathbf{0 . 8 5}$ & 1,00 & ArsenalC12 & 0,77 & 0,7 & 1,00 \\
\hline Rangers $\mathrm{C08}$ & 0.85 & 0,98 & 0.86 & OlympiacosC1 & 0,77 & 0,8 & 0,95 \\
\hline LyonC0 & 0,85 & 0,86 & 0,9 & LyonC07 & 0,77 & 0,8 & 0,94 \\
\hline CSKA MoscowC07 & $\mathbf{0 . 8 5}$ & 0,96 & 0.88 & Standard & 0,77 & 0,9 & 0,79 \\
\hline Unirea UrziceniC10 & 0,84 & 1,00 & 0,8 & JuventusC0 & 0,77 & 0,7 & 0,98 \\
\hline JuventusC1 & 0,84 & 0,98 & 0,8 & Bayern & 0,77 & 0,8 & 0,96 \\
\hline FiorentinaC1 & 0,83 & 0,89 & 0,9 & $\mathrm{AjaxC} 0$ & 0,77 & 0,8 & 0,95 \\
\hline ArsenalC05 & 0,83 & 0,87 & 0,9 & BesiktasC0 & 0,76 & 0,9 & 0,81 \\
\hline RangersC11 & $\mathbf{0 . 8 3}$ & $\mathbf{0 , 9 2}$ & $\mathbf{0 . 9 0}$ & LyonC09 & 0,76 & 0,8 & 0,92 \\
\hline Club & $\overline{0,83}$ & $\overline{0,99}$ & 0,8 & MonacoC05 & 0,76 & 0,8 & 0,89 \\
\hline AEKC0 & 0,83 & 0,89 & 0,9 & Steaua BucarestC07 & 0,76 & 0,9 & 0,81 \\
\hline BarcelonaC0 & 0,82 & 0,84 & 0,9 & Dynamo & 0,76 & 0,9 & 0,83 \\
\hline
\end{tabular}


Vol. 4, No. 4 Zambom-Ferraresi et al.: Sports Results Measurement and Efficiency...

\begin{tabular}{|c|c|c|c|c|c|c|c|}
\hline DMU & TE & PTE & SE & DMU & TE & PTE & SE \\
\hline Rubin KazanC11 & 0,76 & 0,84 & 0,91 & InternazionaleC 12 & 0,71 & 0,75 & 0,95 \\
\hline RomaC05 & 0,76 & 1,00 & 0,76 & ChelseaC07 & 0,71 & 0,73 & 0,97 \\
\hline Bayer LeverkusenC12 & 0,76 & 0,76 & 1,00 & LilleC07 & 0,71 & 0,77 & 0,92 \\
\hline StuttgartC10 & 0,76 & 0,79 & 0,96 & Shakhtar DonetskC09 & 0,71 & 0,86 & 0,82 \\
\hline PanathinaikosC09 & 0,75 & 0,79 & 0,96 & Hapoel Tel-AvivC11 & 0,71 & 0,80 & 0,89 \\
\hline RomaC08 & 0,75 & 0,77 & 0,98 & BarcelonaC07 & 0,71 & 0,74 & 0,95 \\
\hline CopenhaguenC11 & 0,75 & 0,79 & 0,95 & BenficaC06 & 0,71 & 0,76 & 0,93 \\
\hline CopenhaguenC07 & 0,75 & 0,92 & 0,81 & Sporting LisboaC08 & 0,71 & 0,79 & 0,89 \\
\hline NordsjaellandC13 & 0,75 & 0,86 & 0,86 & Paris Saint-GermainC13 & 0,71 & 0,72 & 0,98 \\
\hline LiverpoolC09 & 0,75 & 0,77 & 0,97 & Manchester UnitedC10 & 0,71 & 0,72 & 0,98 \\
\hline PanathinaikosC11 & 0,75 & 0,95 & 0,78 & ChelseaC06 & 0,70 & 0,77 & 0,92 \\
\hline Shakhtar DonetskC11 & 0,75 & 0,76 & 0,98 & KRC GenkC12 & 0,70 & 0,86 & 0,82 \\
\hline Real MadridC05 & 0,75 & 0,76 & 0,97 & ArsenalC08 & 0,70 & 0,72 & 0,98 \\
\hline Dynamo KyivC10 & 0,74 & 0,95 & 0,78 & MilanC08 & 0,70 & 0,75 & 0,93 \\
\hline InternazionaleC07 & 0,74 & 0,83 & 0,89 & CSKA MoscowC12 & 0,70 & 0,72 & 0,97 \\
\hline JuventusC06 & 0,74 & 0,76 & 0,98 & CelticC09 & 0,70 & 0,87 & 0,81 \\
\hline Real MadridC07 & 0,74 & 0,77 & 0,96 & ArsenalC07 & 0,70 & 0,72 & 0,97 \\
\hline RomaC07 & 0,74 & 0,77 & 0,96 & LyonC08 & 0,70 & 0,77 & 0,91 \\
\hline TottenhamC11 & 0,74 & 0,75 & 0,99 & LiverpoolC06 & 0,70 & 0,78 & 0,89 \\
\hline ChelseaC09 & 0,74 & 0,75 & 0,99 & CFR ClujC11 & 0,70 & 0,87 & 0,80 \\
\hline OlympiacosC06 & 0,74 & 0,78 & 0,95 & Debreceni VSCC10 & 0,70 & 0,98 & 0,71 \\
\hline Bayer LeverkusenC05 & 0,74 & 0,76 & 0,97 & CelticC05 & 0,70 & 0,87 & 0,80 \\
\hline AalborgC09 & 0,74 & 0,89 & 0,83 & BetisC06 & 0,70 & 0,76 & 0,91 \\
\hline PortoC10 & 0,73 & 0,80 & 0,92 & CSKA MoscowC10 & 0,70 & 0,76 & 0,92 \\
\hline ValenciaC07 & 0,73 & 0,78 & 0,94 & FiorentinaC09 & 0,70 & 0,86 & 0,80 \\
\hline TrabzonporC12 & 0,73 & 0,81 & 0,90 & VillarrealC09 & 0,69 & 0,71 & 0,98 \\
\hline UdineseC06 & 0,73 & 0,90 & 0,82 & ArsenalC10 & 0,69 & 0,73 & 0,95 \\
\hline BenficaC13 & 0,73 & 0,83 & 0,88 & Spartak MoscowC07 & 0,69 & 0,78 & 0,88 \\
\hline RomaC09 & 0,73 & 0,83 & 0,88 & BenficaC12 & 0,69 & 0,69 & 1,00 \\
\hline PSV EindhovenC05 & 0,73 & 0,73 & 0,99 & MarseilleC10 & 0,69 & 0,85 & 0,81 \\
\hline Viktoria PlzenC12 & 0,72 & 0,87 & 0,83 & Real MadridC10 & 0,68 & 0,71 & 0,96 \\
\hline Real MadridC06 & 0,72 & 0,74 & 0,97 & WolfsburgC10 & 0,68 & 0,85 & 0,80 \\
\hline ThunC06 & 0,72 & 0,91 & 0,79 & PSV EindhovenC07 & 0,68 & 0,73 & 0,94 \\
\hline PortoC09 & 0,72 & 0,78 & 0,93 & ArsenalC09 & 0,68 & 0,69 & 0,99 \\
\hline PortoC07 & 0,72 & 0,81 & 0,89 & RosenborgC08 & 0,68 & 0,85 & 0,80 \\
\hline BarcelonaC10 & 0,72 & 0,73 & 0,99 & AZ AlkmaarC10 & 0,68 & 0,83 & 0,82 \\
\hline PortoC08 & 0,72 & 0,77 & 0,93 & Shakhtar DonetskC07 & 0,68 & 0,80 & 0,84 \\
\hline BaselC12 & 0,72 & 0,76 & 0,94 & FenerbahçeC06 & 0,68 & 0,77 & 0,88 \\
\hline MarseilleC12 & 0,72 & 0,72 & 1,00 & LazioC08 & 0,68 & 0,85 & 0,79 \\
\hline LiverpoolC08 & 0,72 & 0,73 & 0,99 & BordeauxC07 & 0,68 & 0,82 & 0,82 \\
\hline Manchester UnitedC07 & 0,72 & 0,73 & 0,98 & Werder BremenC09 & 0,68 & 0,73 & 0,92 \\
\hline Bayern MunichC09 & 0,72 & 0,75 & 0,96 & BATE BorisovC13 & 0,68 & 0,78 & 0,87 \\
\hline BarcelonaC13 & 0,71 & 0,72 & 0,99 & Dynamo KyivC13 & 0,67 & 0,78 & 0,86 \\
\hline Bayern MunichC07 & 0,71 & 0,73 & 0,98 & RosenborgC06 & 0,67 & 0,88 & 0,76 \\
\hline BATE BorisovC09 & 0,71 & 0,90 & 0,79 & SevillaC08 & 0,67 & 0,75 & 0,90 \\
\hline BordeauxC09 & 0,71 & 0,79 & 0,91 & JuventusC13 & 0,67 & 0,68 & 0,99 \\
\hline FenerbahçeC08 & 0,71 & 0,73 & 0,97 & ArsenalC11 & 0,67 & 0,71 & 0,95 \\
\hline Werder BremenC07 & 0,71 & 0,86 & 0,83 & Real MadridC11 & 0,67 & 0,68 & 0,99 \\
\hline
\end{tabular}




\begin{tabular}{|c|c|c|c|c|c|c|c|}
\hline DMU & TE & PTE & SE & DMU & TE & PTE & SE \\
\hline InternazionaleC09 & 0,67 & 0,71 & 0,94 & Dinamo ZagrebC12 & 0,62 & 0,90 & 0,69 \\
\hline PSV EindhovenC08 & 0,67 & 0,82 & 0,81 & Steaua BucarestC09 & 0,62 & 0,85 & 0,73 \\
\hline AnderlechtC06 & 0,67 & 0,87 & 0,77 & BenficaC11 & 0,62 & 0,78 & 0,79 \\
\hline MilanC13 & 0,67 & 0,70 & 0,95 & Spartak MoscowC13 & 0,61 & 0,80 & 0,77 \\
\hline Werder BremenC06 & 0,66 & 0,74 & 0,89 & Dinamo ZagrebC13 & 0,61 & 0,81 & 0,75 \\
\hline ChelseaC13 & 0,66 & 0,77 & 0,86 & ZenitC09 & 0,61 & 0,74 & 0,82 \\
\hline Schalke 04C08 & 0,66 & 0,71 & 0,93 & OlympiacosC07 & 0,61 & 0,81 & 0,75 \\
\hline MarseilleC08 & 0,66 & 0,78 & 0,85 & Sparta PragueC05 & 0,60 & 0,82 & 0,74 \\
\hline Real MadridC08 & 0,66 & 0,68 & 0,97 & Schalke04C13 & 0,60 & 0,65 & 0,94 \\
\hline MilanC12 & 0,66 & 0,69 & 0,96 & ZenitC13 & 0,59 & 0,70 & 0,85 \\
\hline Atlético MadridC10 & 0,65 & 0,82 & 0,80 & MŠK ŽilinaC11 & 0,59 & 0,88 & 0,68 \\
\hline ChelseaC11 & 0,65 & 0,68 & 0,96 & Otelul GalatiC12 & 0,59 & 0,84 & 0,71 \\
\hline MilanC11 & 0,65 & 0,71 & 0,91 & Shakhtar DonetskC13 & 0,59 & 0,67 & 0,89 \\
\hline BenficaC08 & 0,65 & 0,81 & 0,81 & Dynamo KyivC07 & 0,59 & 0,83 & 0,71 \\
\hline Deportivo La CoruñaC05 & 0,65 & 0,81 & 0,80 & PortoC12 & 0,59 & 0,70 & 0,85 \\
\hline LilleC06 & 0,65 & 0,83 & 0,78 & AnderlechtC05 & 0,59 & 0,73 & 0,81 \\
\hline Sparta PragueC06 & 0,65 & 0,87 & 0,75 & InternazionaleC11 & 0,59 & 0,64 & 0,92 \\
\hline RangersC 10 & 0,65 & 0,87 & 0,74 & HamburgC07 & 0,59 & 0,79 & 0,74 \\
\hline RosenborgC05 & 0,65 & 0,80 & 0,81 & AjaxC11 & 0,59 & 0,72 & 0,82 \\
\hline BarcelonaC12 & 0,65 & 0,65 & 0,99 & AjaxC12 & 0,58 & 0,67 & 0,87 \\
\hline GalatasarayC07 & 0,65 & 0,82 & 0,78 & Werder BremenC11 & 0,58 & 0,73 & 0,79 \\
\hline Real MadridC09 & 0,65 & 0,69 & 0,94 & Werder BremenC08 & 0,57 & 0,76 & 0,76 \\
\hline Manchester CityC12 & 0,64 & 0,71 & 0,91 & FenerbahçeC09 & 0,57 & 0,74 & 0,77 \\
\hline Manchester UnitedC06 & 0,64 & 0,80 & 0,80 & LyonC11 & 0,57 & 0,62 & 0,92 \\
\hline Sporting LisboaC07 & 0,64 & 0,80 & 0,81 & LevskiC07 & 0,57 & 0,77 & 0,74 \\
\hline RomaC11 & 0,64 & 0,73 & 0,88 & Dynamo KyivC08 & 0,57 & 0,79 & 0,72 \\
\hline BaselC09 & 0,64 & 0,89 & 0,72 & StuttgartC08 & 0,56 & 0,73 & 0,77 \\
\hline Spartak MoscowC11 & 0,64 & 0,74 & 0,86 & MarseilleC09 & 0,56 & 0,74 & 0,76 \\
\hline Maccabi HaifaC10 & 0,64 & 0,89 & 0,72 & BursasporC11 & 0,56 & 0,77 & 0,73 \\
\hline Bayern MunichC12 & 0,64 & 0,64 & 1,00 & ValenciaC11 & 0,56 & 0,63 & 0,89 \\
\hline BATE BorisovC12 & 0,64 & 0,84 & 0,76 & Manchester UnitedC12 & 0,56 & 0,68 & 0,82 \\
\hline CFR ClujC09 & 0,64 & 0,84 & 0,76 & Steaua BucarestC08 & 0,55 & 0,76 & 0,72 \\
\hline AnderlechtC07 & 0,63 & 0,82 & 0,77 & VillarrealC12 & 0,55 & 0,80 & 0,69 \\
\hline PanathinaikosC06 & 0,63 & 0,82 & 0,78 & Rapid WienC06 & 0,55 & 0,82 & 0,67 \\
\hline Bayern MunichC11 & 0,63 & 0,64 & 0,98 & ValenciaC12 & 0,54 & 0,65 & 0,83 \\
\hline MarseilleC11 & 0,63 & 0,67 & 0,94 & Manchester CityC13 & 0,54 & 0,67 & 0,81 \\
\hline BesiktasC10 & 0,63 & 0,82 & 0,77 & LilleC13 & 0,54 & 0,75 & 0,72 \\
\hline ValenciaC08 & 0,63 & 0,75 & 0,84 & LilleC12 & 0,54 & 0,68 & 0,79 \\
\hline AnderlechtC13 & 0,62 & 0,74 & 0,84 & CSKA MoscowC08 & 0,54 & 0,79 & 0,68 \\
\hline ZenitC12 & 0,62 & 0,65 & 0,96 & BaselC11 & 0,54 & 0,68 & 0,79 \\
\hline GalatasarayC13 & 0,62 & 0,65 & 0,95 & Borussia DortmundC12 & 0,54 & 0,67 & 0,81 \\
\hline AJ AuxerreC11 & 0,62 & 0,80 & 0,78 & PSV EindhovenC09 & 0,53 & 0,71 & 0,75 \\
\hline Manchester UnitedC13 & 0,62 & 0,69 & 0,90 & LyonC12 & 0,53 & 0,60 & 0,88 \\
\hline Real MadridC13 & 0,62 & 0,63 & 0,98 & AjaxC13 & 0,53 & 0,65 & 0,81 \\
\hline Real MadridC12 & 0,62 & 0,63 & 0,99 & Shakhtar DonetskC12 & 0,51 & 0,65 & 0,78 \\
\hline TwenteC11 & 0,62 & 0,74 & 0,83 & PartizanC11 & 0,51 & 0,73 & 0,70 \\
\hline Shakhtar DonetskC08 & 0,62 & 0,75 & 0,82 & MontpellierC13 & 0,51 & 0,67 & 0,76 \\
\hline PortoC13 & 0,62 & 0,66 & 0,93 & Sporting BragaC13 & 0,51 & 0,69 & 0,73 \\
\hline
\end{tabular}

Notes: DMU: Decision Making Unit; TE: Technical Efficiency; PTE: Pure Technical Efficiency; SE: Scale Efficiency; Italic type: champion of the indicated year; and Boldface and underline: technical efficient in a seasonal basis (TE, PTE and $\mathrm{SE}=1$ ) 
In terms of technical efficiency, an analysis of results along the seasons studied in this paper, it can be seen a high degree of inefficiency in the UCL. The clubs that have been efficient are the following: APOEL (2011/12 season), CFR Cluj (2012/13 season), Internazionale (2009/10 season) and Liverpool (2004/05 season).

Table 3. Technical Efficient Teams in a Seasonal Analysis

\begin{tabular}{llll}
\hline DMU & Season & DMU & Season \\
\hline Anorthosis & $2008 / 09$ & Juventus & $2008 / 09$ \\
APOEL & $2011 / 12$ & Liverpool & $2004 / 05$ \\
APOEL & $2009 / 10$ & Liverpool & $2006 / 07$ \\
Arsenal & $2005 / 06$ & Manchester United & $2007 / 08$ \\
Arsenal & $2012 / 13$ & Manchester United & $2010 / 11$ \\
Barcelona & $2005 / 06$ & Milan & $2006 / 07$ \\
Barcelona & $2008 / 09$ & PSV & $2005 / 06$ \\
Barcelona & $2010 / 11$ & Rangers & $2007 / 08$ \\
Bayern Munich & $2012 / 13$ & Rangers & $2010 / 11$ \\
CFR Cluj & $2012 / 13$ & Schalke 04 & $2010 / 11$ \\
Chelsea & $2011 / 12$ & Slavia Praha & $2007 / 08$ \\
CSKA Moskva & $2006 / 07$ & Sporting Lisboa & $2008 / 09$ \\
Internazionale & $2009 / 10$ & Valencia & $2012 / 13$ \\
\hline
\end{tabular}

Note: Italic type: champion of the indicated season.

In Table 3, it is shown the teams that are efficient in the season-by-season analysis. We can observe that the number of efficient clubs decreases from twentysix clubs in a season-by-season analysis (approximately 9\% of the sample) to four clubs in the entire period, less than $1.4 \%$ of total clubs. As efficiency is a relative measure that depends on the units under comparison, we should expect that the results could change in an overall efficiency study. In our case, as was expected from the characteristics of the methodology, the technical efficiency reduces in general, and the number of efficient units decreases drastically. Many DMUs, which were efficient on a seasonal basis, stop being so.

Comparing efficiency results and sport results, it is possible to highlight that only two of the four efficient clubs went on to win the Championships. Conversely, seven of the nine winning clubs were calculated as being not efficient. In the calculations for season by season, it could be argued that the teams that won the UCL in some of the seasons did so without wasting resources, in contrast with the resources and tactics followed by their opponents. Nevertheless, when all the seasons were considered simultaneously, the range of potential combinations of resources and tactics was considerably higher. Hence, the winning club in the UCL that was efficient in a season-by-season approach but inefficient in the pooled analysis, could obtain the same sporting 
result with fewer resources. Consequently, these teams could not be considered efficient in terms of the analysis combining all the seasons.

In the decomposition of the technical efficiency rate into pure technical efficiency and scale efficiency we can detect the potential sources of inefficiency. In Table 2, it is shown that teams are inefficient not only because they clearly wasted resources but also because they have chosen the wrong tactics. Only $2.7 \%$ (eight teams) of the total sample displayed efficiency in terms of pure technical efficiency, while 5.2\% (15 teams) demonstrated efficiency in terms of scale efficiency. Thus, it seems that waste of resources (lack of pure technical efficiency) is a greater source of inefficiency than is the use of incorrect tactics (lack of scale efficiency).

By clubs, it is possible to detect some inefficient clubs which have made correct use of sporting resources (Olympiacos 2004/05, Maccabi Tel Aviv 2004/05, Dynamo Kyiv 2004/05, Roma 2004/05, Manchester United 2007/08, Anorthosis 2008/09, APOEL 2009/10 and Unirea Urziceni 2009/10). Also, there are some teams which have chosen the correct sporting tactics (Milan 2004/05, Arsenal 2005/06 and 2011/12, Milan 2006/07, Liverpool 2006/07, Chelsea 2007/08, Manchester United 2008/09 and 2010/11, Bayern Munich 2009/10 and 2012/13, Bayer Leverkusen 2011/12, Marseille 2011/12, Benfica 2011/12, Arsenal 2012/13 and Malaga 2012/13).Concerning efficient teams in the seasonby-season analysis and inefficient teams for the sample as a whole, only Milan in season 2006/07 and Manchester in season 2007/08 showed one type of efficiency when taking into account the whole sample. Milan, showing a scale efficiency of 1 in the panel data analysis, chose correctly sporting tactics to develop a correct use of its sporting resources. Also, Manchester United with a rate of pure technical efficiency of 1 used its sporting resources without a waste of resources.

Finally, from a longitudinal analysis of the evolution of efficiency rates, it is not possible to detect any clear pattern. However, three out of four efficiency clubs have been efficient in the last seasons. Also, the inefficient clubs with values of pure technical efficiency of one are concentred in the first seasons, while the teams with scale efficiencies of 1 are concentred in the last seasons.

\section{Discussion and Conclusions}

In general, in many sports and football, in particular, analysis of how efficiently a club uses its resources is really important to evaluate its performance. Among the different tools widely applied in the literature to measure efficiency, we have opted for DEA methodology. Cooper et al. (2007) highlight its ability to identify the benchmark members of the efficient set used to effect these evaluations and to identify the sources (and amounts) of inefficiency.

In the present paper, we have applied this methodology to the best clubs in Europe, 94 different clubs that played in the Champions League in nine seasons (2004/05-2012/13). The use of a great number of seasons, allows us to provide interesting conclusions for each season as well as for the whole period of time. 
In this paper we measured the level of efficiency of UCL clubs as well as their main sources of inefficiency.

We show that there is a high degree of inefficiency in the UCL: the number of efficient clubs is only $1.4 \%$ of the total sample. Moreover, over the nine seasons analysed, no club managed to maintain technical efficiency. Thus, our hypothesis of an expected low level of efficiency among the most important European football clubs has been confirmed. This result is in accordance with previous empirical evidence about efficiency in the UCL (Espitia-Escuer and GarcíaCebrián 2010, Papahristodoulou 2006). Our finding implies that it is very difficult for clubs to sustain their efficiency in what is the most competitive European football tournament.

Furthermore, two different inefficiency sources were detected in our study. The first inefficiency source was associated with the choice of inappropriate sporting tactics. The second, related to a clear waste of resources. The results showed that only $2.7 \%$ of clubs $(n=8)$ made correct use of their resources, while $5.2 \%(\mathrm{n}=15)$ employed adequate sports tactics. Hence, it appears that waste of resources (lack of pure technical efficiency) is a greater source of inefficiency than is the use of incorrect tactics (lack of scale efficiency).

Differences in measures, methodology and so on, that were emphasised in the literature review, make it difficult to compare these findings with those of previous studies. With particular reference to our new output, differences between our study and previous studies of the UCL (i.e. Espitia-Escuer and García-Cebrián 2010, Papahristodoulou 2006) were expected and confirmed. Anyway, the new output measure proposed seems to be suitable for representing reliably the sports results archived by the football clubs that play UCL. This new measure could allocate more real values to the sports results. This is easiest to comprise considering that with the output in previous studies, between the champion and the runners-up team, and among the sixteen teams which did not qualify to the knockout phase, there were no differences in outcomes. Then, many of the runners-up and teams that did not qualify to the knockout phase, which theoretically had a very poor sporting performance, were classified as efficient in previous studies. The use of the coefficients applied by UEFA from UCL revenue distribution will be helpful in future research.

In spite of the fact that it has not been possible to establish a pattern in the evolution of efficiency values shown by the teams, a temporal concentration of values for one was detected. In other words, when the whole sample was used in the calculations, the efficient clubs are those participating in the former seasons in our sample, as did the clubs who demonstrated only scale efficiency (whose sporting tactics were correct). On the other hand, inefficient clubs with a pure technical efficiency ratio equal to one (i.e. teams with incorrect sporting tactics) were concentrated in the first season of the whole sample. These results might therefore suggest that the teams changed and improved their sporting tactics from an efficiency point of view. From this longitudinal perspective, this result contrasts with that found by Espitia-Escuer and García-Cebrián (2010), neither of whom detected changes in technology or tactics in the period analysed. 
A further detailed analysis of this situation is beyond the scope of this paper but is of interest to future research.

From a managerial perspective, some implications and managerial recommendations can be highlighted. It could be argued that, from the perspective of pure technical efficiency, inefficient clubs should deploy their resources better, i.e. use fewer plays in order to obtain the same results during games. In addition, when forming a new squad for the future, clubs should search for and purchase players with specific characteristics and efficient individual performances, in order to improve results. From the perspective of scale efficiency, inefficient clubs should change their tactics. First, when forming a new squad they should search for players who make few mistakes when playing. Second, it could be argued that they should imitate and apply the same sports tactics that the most efficient clubs employ. Nevertheless, one could also argue against this managerial implication, in that it is precisely by using tactics that are different from those of opposing teams that one can gain sporting success. In this regard, it should be noted that the approach applied in this paper was that of efficiency, namely, the consumption of resources without waste. The evaluation of tactics in accordance with the sports results of teams also includes an assessment of effectiveness. Furthermore, it has been shown in the present paper that a championship winner is not always an efficient team, it is just less inefficient than the runnerup, perhaps.

\section{Acknowledgments}

Our heartfelt thanks to Opta Sports who generously provided the sports data used in this study. Opta Sports (www.optasports.es) is a company with one of the largest sports databases of European football.

Fabíola Zambom Ferraresi was supported by a pre-doctoral fellowship from the Public University of Navarra.

\section{References}

Asmild M, Paradi JC, Aggarwall V, Schaffnit C (2004) Combining DEA window analysis with the Malmquist index approach in a study of the Canadian banking industry. Journal of Productivity Analysis 21(1): 67-89.

Barros CP, Douvis J (2009) Comparative analysis of football efficiency among two small European countries: Portugal and Greece. International Journal of Sport Management and Marketing 6(2): 183-199.

Barros CP, Garcia-del-Barrio P (2008) Efficiency measurement of the English football Premier League with a random frontier model. Economic Modelling 25(5): 9941002.

Barros CP, Leach S (2006) Performance evaluation of the English Premier Football League with data envelopment analysis. Applied Economics 38(12): 1449-1458.

Banker RD, Charnes A, Cooper WW (1984) Some models for estimating technical and scale inefficiencies in data envelopment analysis. Management science 30(9): 1078-1092. 
Boscá JE, Liern V, Martínez A, Sala R (2009) Increasing offensive or defensive efficiency? An analysis of Italian and Spanish football. Omega 37(1): 63-78.

Carmichael F, Thomas D (1995) Production and efficiency in team sports: an investigation of rugby league football. Applied Economics. 27(9): 859-869.

Carmichael F, Thomas D, Ward R (2000) Team Performance: The Case of English Premiership Football. Managerial and Decision Economics 21(1): 31-45.

Charnes A, Cooper WW, Rhodes E (1978) Measuring the efficiency of decision making units. European Journal of Operational Research 2(6): 429-444.

Cooper WW, Seiford LM, Tone K (2007) Data envelopment analysis: a comprehensive text with models, applications, references and DEA-Solver software. New York, NY: Springer.

Cullinane K, Wang TF (2006) Data envelopment analysis (DEA) and improving container port efficiency. Research in Transportation Economics 17: 517-566.

Dawson P, Dobson S, Gerrard B (2000) Stochastic frontiers and the temporal structure of managerial efficiency in English soccer. Journal of Sports Economics 1(4): 341362 .

Debreu G (1951) The coefficient of resource utilization. Econometrica 19(1): 273-292.

Deloitte (2017) Annual Review of Football Finance 2017. Retrieved from https://goo. gl/T58dsy. [Accessed 6 September 2017].

Espitia-Escuer M, García-Cebrián LI (2004) Measuring the Efficiency of Spanish FirstDivision Soccer Teams. Journal of Sports Economics 5(4): 329-346.

Espitia-Escuer M, García-Cebrián LI (2006) Performance in sports teams: results and potential in the professional soccer league in Spain. Management Decision 44(8): 1020-1030.

Espitia-Escuer M, García-Cebrián LI (2010) Measurement of the efficiency of football teams in the champions league. Managerial and Decision Economics 31(6): 373386.

Farrell MJ (1957) The Measurement of Productive Efficiency. Journal of the Royal Statistical Society, Series A (General) 120(3): 253-290.

Førsund FR, Lovell CAK, Schmidt P (1980) A survey of Frontier Production Functions and of their relationship to efficiency measurement. Journal of Econometrics, 13(1): $5-25$.

Garcia-del-Barrio P, Szymanski S (2009) Goal! Profit Maximization versus Win Maximization in Soccer. Review of Industrial Organization 34(1): 45-68.

Gerrard B (2010) Analysing sporting efficiency using standardised win cost: Evidence from the FA Premier League, 1995 - 2007. International Journal of Sports Science and Coaching 5(1): 13-35.

Giner-Vicente C, Muñoz-Porcar A (2008) ¿Son los clubes de fútbol eficientes? Aplicación del análisis DEA a los equipos de la Liga Profesional de Fútbol de España. Universia Business Review 17: 12-25.

González-Gómez F, Picazo-Tadeo AJ (2010) Can We Be Satisfied With Our Football Team? Evidence from Spanish Professional Football. Journal of Sports Economics 11(4): 418-442.

Haas DJ (2003) Productive Efficiency of English Football Teams - A Data Envelopment Analysis Approach. Managerial and Decision Economics 24(5): 403-410.

Haas D J (2003a) Technical Efficiency in the Major League Soccer. Journal of Sports Economics 4(3): 203-215.

Horowitz I (2007) If you play well they will come? and vice versa: bidirectional causality in major-league baseball. Managerial and Decision Economics 28(2): 93-105. 
Lago-Peñas C, Lago-Ballesteros J, Rey E (2011) Differences in performance indicators between winning and losing teams in the UEFA Champions League. Journal of Human Kinetics 27(1): 135-146.

Palacios-Huerta I (2004) Structural changes during a century of the world's most popular sport. Statistical Methods and Applications 13(2): 241-258.

Papahristodoulou C (2006) Team Performance in UEFA Champions League 2005-06. Working paper No. 138, MPRA Paper, University Library of Munich, Munich, 05 September.

Ribeiro AS, Lima F (2012) Portuguese football league efficiency and players' wages. Applied Economics Letters 19(6): 599-602.

Schofield JA (1988) Production functions in the sports industry: an empirical analysis of professional cricket. Applied Economics 20(2): 177-193.

Scully GW (1974) Pay and Performance in Major League Baseball. The American Economic Review 64(6): 915-930.

Torres-Dávila CG, García-Cebrián LI (2012) Eficiencia y resultados deportivos: aplicación a la liga mexicana de fútbol. Movimiento humano 3: 61-76.

Tulkens H, Vanden Eeckaut P (1995) Non-parametric efficiency, progress and regress measures for panel data: Methodological aspects. European Journal of Operational Research 80: 474-499.

UEFA (2015) The European Club Footballing Landscape: Club Licensing Benchmarking Report Financial Year 2015. Retrieved from https://goo.gl/a7bnVW. [Accessed 6 September 2017].

UEFA (2017) UEFA Champions League continues impressive digital growth by Andy James \& Nina Tsimpouli. Retrieved from https://goo.gl/Jc4GBK. [Accessed 8 September 2017]. 
